\title{
In Dirac's shadow
}

\author{
R.B. Jones
}

IT IS a malicious fate that sends a new book on quantum mechanics to be reviewed beside that of Dirac. It places an inhuman burden of restraint on the reviewer, whose first thoughts of Decline and Fall seem to require the pen of Gibbon or perhaps rather Evelyn Waugh. Dirac's classic textbook, The Principles of Quantum Mechanics, first appeared in 1930 but it remains incomparably the best book on the fundamentals of quantum theory. Here it is freshly reprinted as a paperback from its hardbound fourth edition, incorporating the revisions of 1967 on quantum field theory.

For those not yet familiar with it, let me say briefly that starting from the clearest of all accounts of the linear structure of quantum mechanics it proceeds to cover all the standard non-relativistic aspects of quantum mechanics other than variational techniques. In addition, the relativistic single electron and the quantum field theory of photons, electrons and positrons are described with masterly clarity and economy. Only the section on field theory may be regarded as provisional or seriously incomplete, since there is no hint of the rich development of quantum field theories since 1967 for the description of leptons and hadrons. All serious students should read this book but it demands unflagging intellectual courage. Moreover, it seems to get better and to yield more insights with increasing experience on the part of the reader. For these reasons almost no lecturer dares to use it as a standard textbook.

It is doubtless for similar reasons that we have, year after year, yet another crop of new books on quantum mechanics for irascible reviewers to condemn. John Martin's Basic Quantum Mechanics is meant as an introductory book for physicists. His approach is modern and non-historical, and from the beginning he banishes classical thinking in favour of the calculus of probability amplitudes. This approach is shown to good effect in his use of the Stern-Gerlach experiment to set up the two-component description of electron spin. He treats the standard solvable problems and then covers timeindependent and time-dependent perturbation theory through the golden rule and the resonance approximation. There is a short account of dipole radiation and finally an introduction to the variational principle. Many familiar topics, however, are missing. There is very little discussion of the uncertainty principle
The Principles of Quantum Mechanics, 4th Edn. By P.A.M. Dirac. Pp.314. Pbk ISBN 0-19-852011-5. (Oxford University Press: 1981.) £7.95, \$29.50. Basic Quantum Mechanics. By J.L. Martin. Pp.241. Hbk ISBN 0-19-851815-3; pbk ISBN 0-19. 851816-1. (Oxford University Press: 1981.) Hbk £17.50, \$39.50; pbk £7.95, \$17.95. Quantum Mechanics. By Hendrik E. Hameka. Pp.387. ISBN 0-471-09223-1. (Wiley: 1981.) £24, \$43.25. Quantum Mechanics For Applied Physics and Engineering. By Albert Thomas Fromhold, Jr. Pp.430. ISBN 0-12-26915-4. (Academic: 1981.) £22.60, \$34.

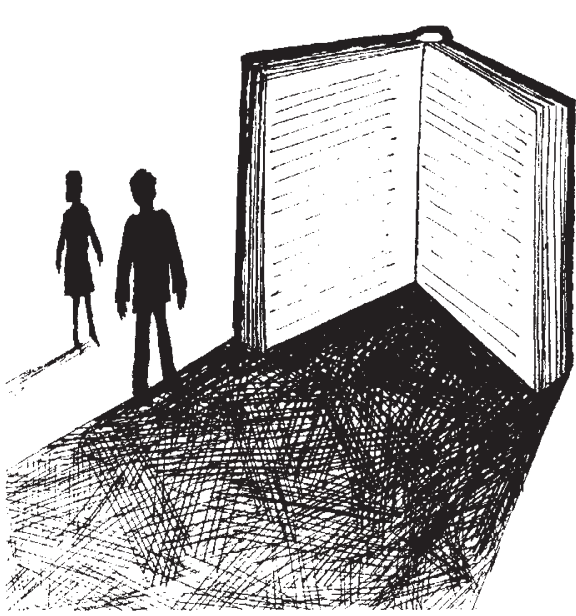

and no mention of the geometrical shape of wave functions or of the link between nodes and quantum numbers. There is no commutation rule treatment of orbital angular momentum eigenvalues, no threedimensional scattering, no exclusion principle. In spite of many such omissions, this is a good, well-written introductory book with a distinctive point of view to justify its existence.

Quantum Mechanics by Hendrik F. Hameka is a book written for chemists. His approach is historical and primarily wave mechanical; he has made the book selfcontained in its mathematical techniques to a degree not necessary for physics students but perhaps helpful to chemists. In this respect he over-emphasizes the role of special functions at the expense of matrix mechanics aspects of the subject. His treatment of the standard solvable examples is undistinctive and for me the book only came to life with the treatment of approximation methods. There is a nice combination of time-independent perturbation theory with variational techniques to include perturbations about approximate eigenfunctions. Timedependent perturbation theory includes an introduction to line widths and lifetimes. There is both a semi-classical and a second quantized treatment of atomic radiation. Spin and the exclusion principles are discussed, with a clear introduction to Slater determinants and Slater orbitals in the Hartree-Fock treatment of manyelectron atoms. There is very little on angular momentum addition and no threedimensional scattering theory. This is a reasonable textbook for a course biased towards atomic structure but, while perhaps an attractive book for chemistry students, it will not be a text of first choice for physicists.

Albert Thomas Fromhold, Jr, calls his book Quantum Mechanics for Applied Physics and Engineering and intends it as a two-year course for technologists working with solid state devices such as tunnel diodes or Josephson junctions. It is, in fact, three books in one for he covers elementary quantum mechanics, statistical mechanics of ideal quantum gases and solid state physics - an ambitious venture which does not succeed to my mind because these three subjects appear in unbalanced and piecemeal form. Thus in the section on quantum mechanics the simple harmonic oscillator and the hydrogen atom are omitted, apart from quoting their energy levels, while endless pages are devoted to one-dimensional tunnelling probabilities for square and trapezoidal barriers in WKB approximation. One learns about the almost free electron model of metals but nothing serious about phonons or magnetic properties of solids. The book is well produced and copiously supplied with problems and illustrations, but I do not think this approach will produce broadly based or coherent understanding. It would not take so much longer to learn these subjects as a physicist would by using the excellent textbooks already available and, incidentally, obtaining a much broader understanding.

One last stone remains to be hurled at each of the three books just considered. The authors treat mathematics as a subject which will terrify or confound their readers. It is in fact the natural language of quantum mechanics, and should be used without comment rather than segregated into separate sections marked "Dangerous".

R.B. Jones is a Lecturer in Physics at Queen Mary College, University of London. 